\title{
Uniportal versus multiportal video-assisted thoracic surgery for lung cancer
}

\author{
Mamdoh Al-Ameri ${ }^{1,2}$, Erik Sachs $^{1,2}$, Ulrik Sartipy ${ }^{1,2}$, Veronica Jackson $^{2}$ \\ ${ }^{1}$ Heart and Vascular Theme, Karolinska University Hospital, Stockholm, Sweden; ${ }^{2}$ Department of Molecular Medicine and Surgery, Karolinska \\ Institutet, Stockholm, Sweden \\ Contributions: (I) Conception and design: All authors; (II) Administrative Support: U Sartipy; (III) Provision of study materials or patients: None; \\ (IV) Collection and assembly of data: All authors; (V) Data analysis and interpretation: All authors; (VI) Manuscript writing: All authors; (VII) Final \\ approval of manuscript: All authors. \\ Correspondence to: Veronica Jackson, MD, PhD. Department of Molecular Medicine and Surgery, Karolinska Institutet, Karolinska University \\ Hospital, SE-17176 Stockholm, Sweden. Email: Veronica.Jackson@ki.se.
}

Background: Video-assisted thoracic surgery (VATS) lobectomy is the recommended surgical approach for patients with stage I lung cancer. Whether a multiportal or a uniportal approach is preferable remains unclear. The aim of this study was to evaluate the safety of implementing uniportal VATS lobectomy into the treatment program of lung cancer patients.

Methods: We used the national quality register for general thoracic surgery in Sweden and included all patients who underwent VATS lobectomy for lung cancer at the Karolinska University Hospital between 2016-2018. Early postoperative complications were compared in patients undergoing uniportal $(n=122)$ and multiportal ( $\mathrm{n}=211$ ) VATS lobectomy for lung cancer. Inverse probability of treatment weighting and standardized mean differences were used to limit differences in baseline characteristics and to assess balance after weighting.

Results: The proportion of uniportal VATS lobectomies increased during the study period and the conversion rates declined significantly. Baseline characteristics were similar in the two groups with the exception of a higher percentage of patients without any comorbidity in the uniportal group (59.8\% vs. 44.5\%, $\mathrm{P}=0.010$ ). After inverse probability of treatment weighting the groups were well balanced. Postoperative complications were rare regardless of surgical approach, $94 \%$ in both groups had no complications. The 30-day mortality and overall survival at 1 year was $0 \%$ and $97 \%$ in the uniportal group, and $0.5 \%$ and $98 \%$ in the multiportal group $(\mathrm{P}=0.71)$. Patients undergoing uniportal VATS lobectomy were discharged directly to home to a higher extent than multiportal VATS patients $(76.2 \%$ vs. $62.1 \%, \mathrm{P}=0.008)$.

Conclusions: We found that uniportal VATS lobectomy was feasible and safe, and might entail advantages in terms of a faster recovery after surgery as compared to multiportal VATS lobectomy in patients with lung cancer.

Keywords: Uniportal; multiportal; video-assisted thoracic surgery (VATS); lobectomy; lung cancer

Submitted May 06, 2019. Accepted for publication Nov 19, 2019.

doi: $10.21037 /$ jtd.2019.12.01

View this article at: http://dx.doi.org/10.21037/jtd.2019.12.01

\section{Introduction}

The adoption of minimally invasive surgical techniques in the management of operable lung cancer is increasing worldwide $(1,2)$. Video-assisted thoracic surgery (VATS) lobectomy with a multiportal approach as treatment for lung cancer was introduced in the early 1990s and the first uniportal VATS lobectomy was performed in 2010 (3-5). Multiportal VATS has been shown not to compromise patient safety nor oncological efficacy in patients with lung cancer (6-9). In addition, VATS lobectomy has been 
associated with fewer perioperative complications, faster recovery, less postoperative pain, and better quality of life when compared with thoracotomy lobectomy (10-13). VATS is now the recommended surgical approach in patients with stage I lung cancer $(1,14,15)$. If the uniportal approach entails any advantages with regards to morbidity and mortality in patients with lung cancer remains to be shown (16-18). Proposed benefits with the uniportal approach include a surgical view and a manual coordination similar to that in open surgery as well as a more favourable ergonomic situation for the surgeons and staff (19-21). This might positively affect the general adoption, and the surgical quality, of minimally invasive lung cancer surgery, however, taking into account that adopting a new surgical technique always entails a learning curve (19). There is still a lack of robust evidence demonstrating clinically significant differences in perioperative outcomes between uniportal and multiportal VATS lobectomy $(17,18,22,23)$. At our institution multiportal VATS lobectomy was successfully implemented as the routine procedure for operable lung cancer in 2012 (7). In 2016 the VATS program was expanded to also include uniportal VATS lobectomy. The aim of this study was to evaluate the safety of implementing uniportal VATS lobectomy into the treatment program of lung cancer patients.

\section{Methods}

The study was approved by the Swedish Ethical Review Authority and the need for informed consent was waived (Dnr: 2019-00964).

\section{Study design}

This was an institutional observational cohort study and the STROBE (The Strengthening the Reporting of Observational Studies in Epidemiology (STROBE) statement) and RECORD (The REporting of studies Conducted using Observational Routinely-collected health Data (RECORD) statement) guidelines for observational studies using routinely collected data were followed $(24,25)$.

\section{Patients and outcomes measures}

We used the national quality register for general thoracic surgery in Sweden (ThoR, http://www.ucr.uu.se/thor) to identify the study population. All patients who underwent
VATS lobectomy for lung cancer between January 1, 2016 and December 31, 2018 at the Karolinska University Hospital and who were 18 years or older were included. Karolinska University Hospital is the single provider of thoracic surgery in the Stockholm County, serving approximately 2.5 million persons ( $25 \%$ of the total Swedish population). In two patients uniportal VATS lobectomy was converted to thoracotomy and they were therefore excluded from the analyses. Uniportal VATS lobectomies that were converted to multiportal VATS $(n=12)$ were analysed as multiportal VATS. The final study population consisted of a total of 333 patients, 122 in the uniportal group and 211 in the multiportal group. The main outcome measures were early postoperative complications as available from the ThoR register, and we also report survival during follow-up.

\section{Definitions}

Comorbidity was defined as major medical conditions requiring ongoing treatment or possibly influencing prognosis. Smoking status was divided into three categories; never, former and current. Never smoker was defined as a person who had never actively smoked. Former smoker was defined as smoking cessation more than one month prior to surgery. Current smoker was defined as actively smoking at the time of surgery or smoking cessation within one month of surgery.

\section{Operative technique}

All patients were consecutively operated on by one of three dedicated general thoracic surgeons although the majority of the uniportal VATS lobectomies were performed by one single surgeon. A multiportal approach was defined as either a three-port anterior approach as previously described $(7,26)$ or a two-port anterior approach where the posterior axillary port described in the three-port approach was excluded. We used a uniportal approach as described and developed by Gonzalez-Rivas et al. $(27,28)$. In brief, the method of general anaesthesia, placement of patient and surgeons, thoracoscope, soft tissue retractor, surgical steps, and postoperative care were the same as for the multiportal approach. A 4-5 cm incision was made in the anterior to mid axillary line usually in the fifth intercostal space. The camera was mostly held in the posterior part of the incision by the assistant surgeon. A $24 \mathrm{~F}$ chest tube was placed in the posterior part of the incision in the majority of cases. 


\section{Statistical analyses}

Baseline characteristics were described as means and standard deviations for continuous variables. Categorical variables were described as frequencies and percentages. Time-to-event was calculated as time in days from the date of surgery until the date of death or January 15, 2019, which was the end of follow-up of vital status. Survival was estimated using the Kaplan-Meier method. Comparisons between the uniport and multiport groups were performed using Fishers exact test in inverse probability of treatment weighted (IPTW) samples where the weights were derived from propensity scores estimated using generalized boosted regression modelling $(29,30)$. The following preoperative variables were used in the estimation of propensity scores: age, sex, body mass index, forced expiratory volume, performance status, ischemic heart disease, hypertension, arrhythmia, prior thoracic surgery, congestive heart failure, diabetes, stroke, chronic kidney disease, smoking status, preoperative radiotherapy, preoperative chemotherapy, pathologic stage. We examined the distribution of weights and found no patients with extreme weights, indicating that trimming was not necessary. Balance between the groups was assessed by standardized mean differences. An absolute standardized difference of $\leq 0.1$ was considered an ideal balance, and $\leq 0.2$ is generally considered an acceptable balance (31). Stata version 15.1 (StataCorp LP, College Station, TX, USA) and R version 3.5.2 (R Foundation for Statistical Computing, Vienna, Austria) were used to perform the statistical analyses.

\section{Results}

\section{Patient characteristics}

Three hundred and thirty-three patients underwent minimally invasive lobectomy due to lung cancer at Karolinska University Hospital between 2016 and 2018. Uniportal VATS lobectomy was completed in 122 (37\%) patients. The mean age was 68 years regardless of surgical approach whereas the percentage of women was slightly higher in the multiportal group (65.9\% vs. 60.7\%). The two groups were similar with regards to baseline characteristics with the exception of the proportion of patients without any comorbidity which was higher in the uniportal group (59.8\% vs. $44.5 \%, \mathrm{P}=0.010)$. Baseline characteristics are shown in Table 1.

After inverse probability of treatment weighting, the distribution of baseline characteristics was well balanced between the two groups. The interested reader can find baseline characteristics and absolute standardized differences before and after inverse probability of treatment weighting in a supplementary appendix online (Table $S 1$ and Figure S1).

\section{Number of operations and conversion rates}

Throughout the study period the proportion of uniportal VATS lobectomies increased from $21 \%$ (21/101) during the first year to $42 \%(43 / 103)$ and $45 \%$ (58/129) for the second and third year, respectively (Figure 1). The conversion rates declined significantly over the years. In total 9\% (12/134) of the intended uniportal VATS lobectomies were converted to a multiportal approach. The majority of the conversions occurred at the beginning of the study period, $28 \%(8 / 29)$ in $2016,2 \%(1 / 44)$ in 2017 , and $5 \%(3 / 61)$ in 2018.

\section{Lymph node sampling and radicality}

In the uniportal group a median of four lymph node stations were sampled (Figure 2 and Table S2). In the multiportal group the number of lymph node stations sampled increased from four to five during the study period. The percentage of patients with 3 or more N2 (mediastinal) lymph node stations sampled, including station 7 (subcarinal), was lower in the uniportal group $(40.2 \%$ vs. $61.6 \%, \mathrm{P}<0.001)$ whereas station 7 was sampled to a high extent in both groups (uniportal 91.8\%, multiportal 87.2\%) (Table 2). Patients without microscopically radical surgery were rare regardless of surgical approach (uniportal 0.8\%, multiportal 1.4\%).

\section{Survival and postoperative events}

The median follow-up time was 1.2 years. The 30-day mortality was $0 \%$ vs. $0.5 \%$, and the overall survival at 1 year was $97 \%$ vs. $98 \%(\mathrm{P}=0.71)$ in the uniportal and the multiportal group, respectively (Table 2 and Figure 3). The analyses of postoperative events and complications were performed in the weighted sample (Table 2). Postoperative complications were rare irrespective of surgical approach and $94 \%$ of the patients in both groups had no complications. The proportion of patients that had the chest drain removed on postoperative day one decreased from 2016 through 2018 and the decline was more pronounced in the uniportal group (Figure 2). The median postoperative hospital stay was similar in both groups and was reduced from four to 3 days over the years. The percentage of 
Table 1 Baseline characteristics in 333 patients who underwent VATS lobectomy for lung cancer at Karolinska University Hospital 2016-2018

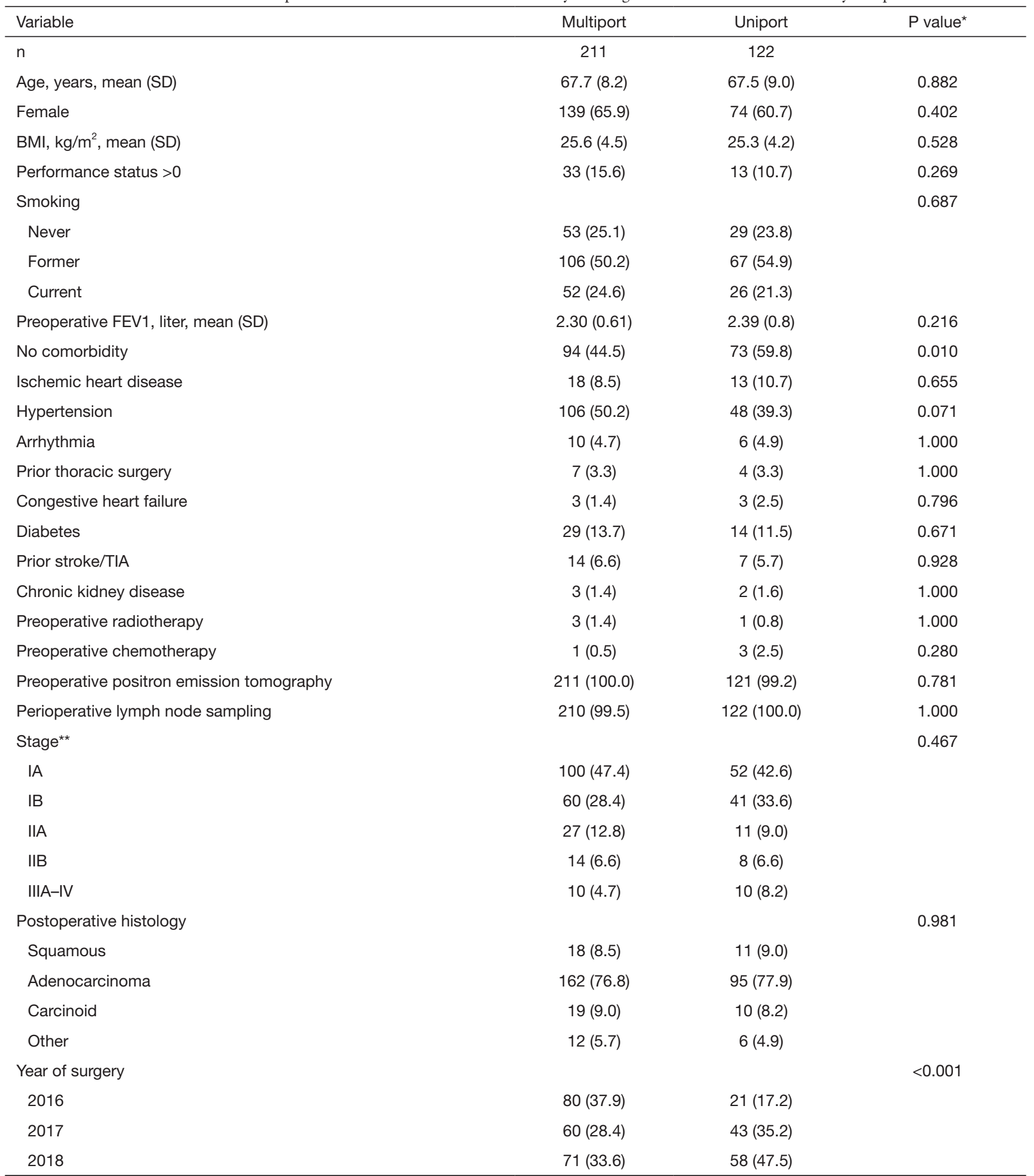

Numbers are $\mathrm{n}(\%)$ unless otherwise noted. *, student's $t$-test or chi-square as appropriate, $\mathrm{P}<0.05$ was considered statistically significant; ${ }^{* *}$, pathologic stage. BMI, body mass index; FEV1, forced expiratory volume in one second; SD, standard deviation; TIA, transient ischemic attack; VATS, video-assisted thoracic surgery. 


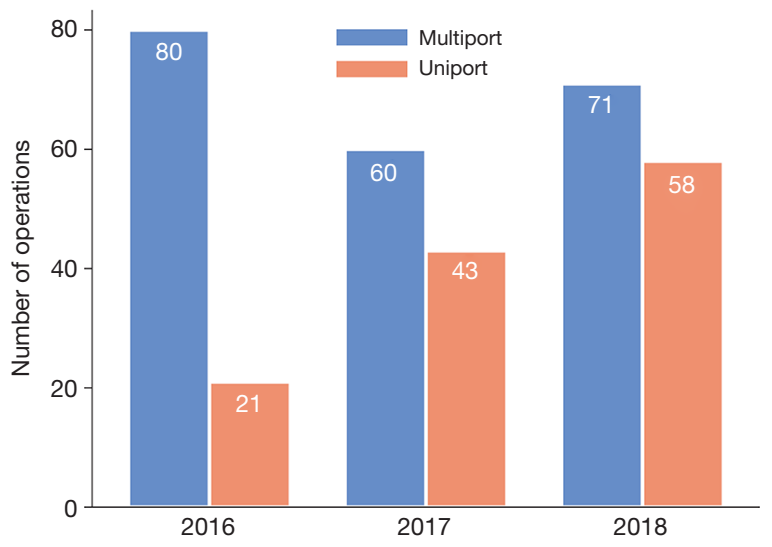

Figure 1 Number of VATS lobectomies performed per year at Karolinska University Hospital 2016-2018. VATS, video-assisted thoracic surgery. patients that were discharged directly to their homes increased throughout the study period and was consistently higher in the uniportal group $(76.2 \%$ vs. $62.1 \%, \mathrm{P}=0.008)$ (Figure 2, Table 2, and Table S2).

\section{Discussion}

The major finding of this study was that uniportal VATS lobectomy for lung cancer patients was feasible and could be safely implemented into the treatment program of lung cancer patients. The postoperative complications were very rare regardless of surgical approach. The percentage of patients with early discharge directly to their homes increased throughout the study period and the increase was consistently more pronounced in the uniportal VATS
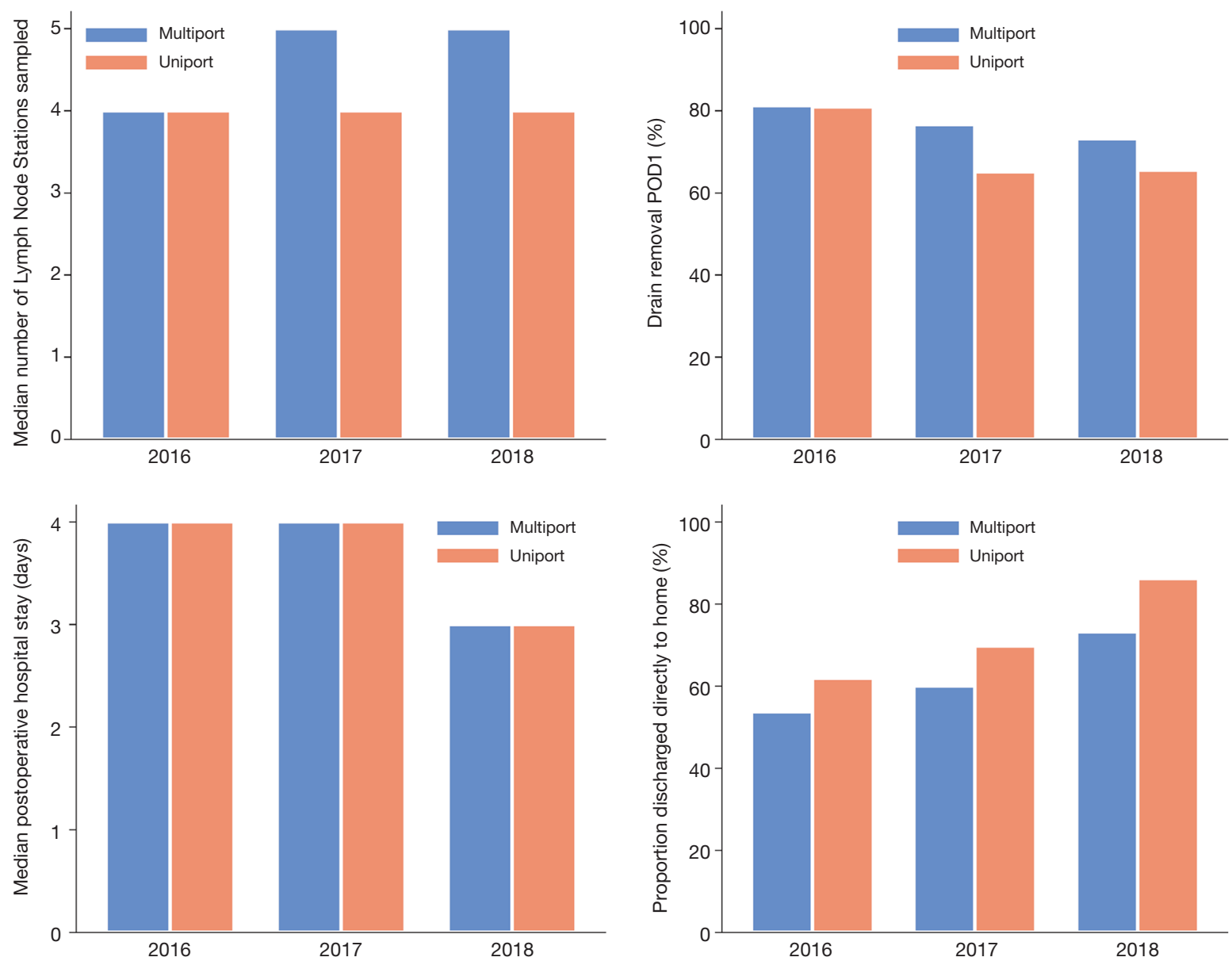

Figure 2 Median number of lymph nodes sampled perioperatively; percentage of patients with chest drain removal on postoperative day one; median postoperative hospital stay in days; proportion of patients discharged directly to home, according to surgical approach. POD 1, postoperative day 1; VATS, video-assisted thoracic surgery. 
Table 2 Postoperative events and complications after VATS lobectomy for lung cancer

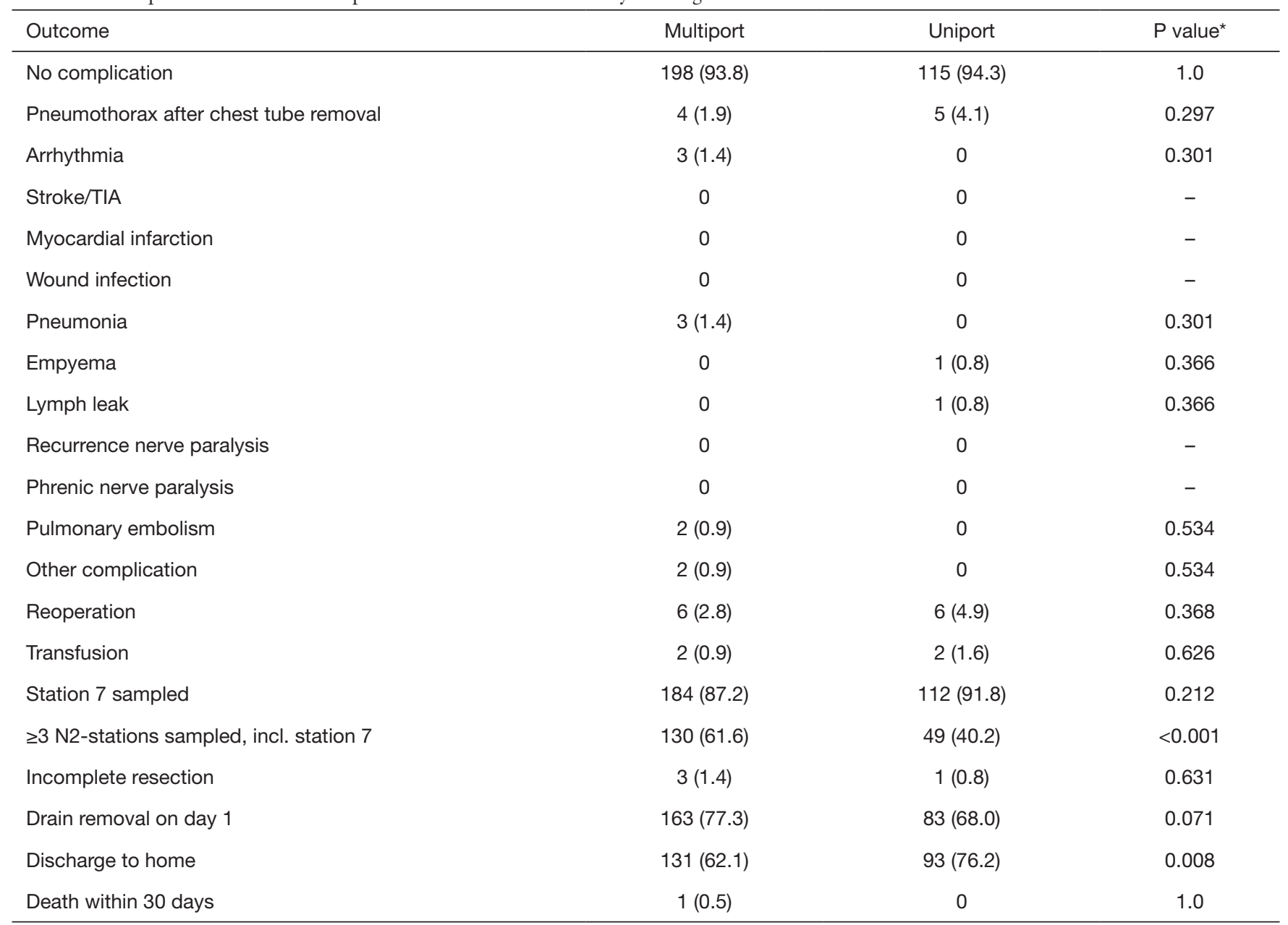

Number are $\mathrm{n}(\%)$ unless otherwise noted. * Fisher's exact test in the inverse probability of treatment weighted sample. $\mathrm{P}<0.05$ was considered statistically significant. TIA, transient ischemic attack; VATS, video-assisted thoracic surgery.

group. Our results suggest that uniportal VATS lobectomy might entail advantages in terms of a faster recovery after surgery.

The increase in the number of uniportal VATS lobectomies performed during the study period, also including patients with advanced pathologic stage, in conjunction with a permanently low complication rate testifies to the feasibility and safety of implementing uniportal VATS lobectomy into the treatment program of lung cancer patients. In addition, the conversion rate to multiportal VATS declined considerably from 28\% in 2016 to $5 \%$ in 2018.

During the study period the median length of stay (LOS) in hospital was reduced from four to three days with no association to surgical approach. This finding might be a consequence of the launch of a treatment protocol for enhanced recovery after thoracic surgery (ERATS) at our institution at the beginning of 2017 , perhaps leading to a measurable effect in 2018. It could be argued that the higher proportion of patients discharged directly to home, as opposed to a rehabilitation centre, also could be related to the implementation of ERATS. In our study a greater proportion of the patients underwent uniportal VATS lobectomy towards the end of the study period when ERATS was more established. However, early discharge directly to home was more frequently seen in the uniportal VATS group than the multiportal group, possibly implying a more favourable recovery process in uniportal VATS patients. To be eligible for discharge directly to home the patients had to be able to independently perform their every-day-activities 


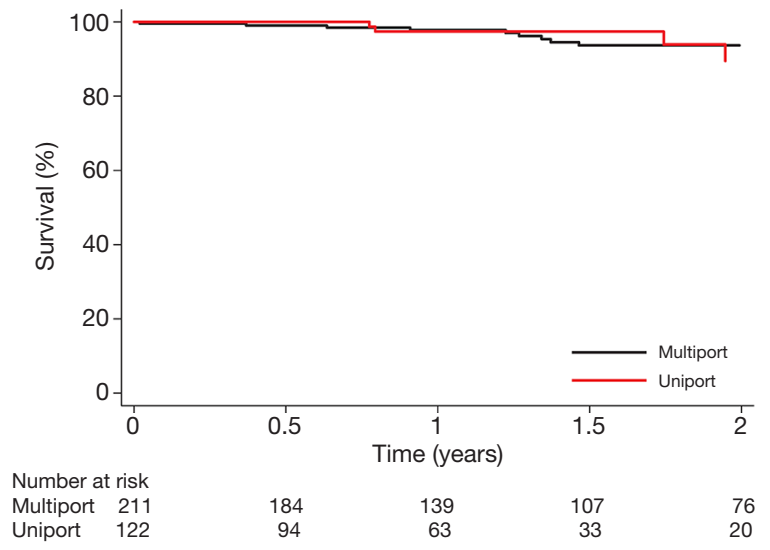

Figure 3 Survival after uniportal or multiportal VATS lobectomy for lung cancer. Survival is plotted against time after surgery and stratified according to surgical approach, uniportal VATS lobectomy (red line) and multiportal VATS lobectomy (black line). VATS, video-assisted thoracic surgery.

and not be dependent on the care of others.

In a recent Dutch national study, von Meyenfeldt et al. found that mean LOS increased by 1.8 days after converted operations (VATS to thoracotomy) and the authors conclude that age, extent of resection, and surgical approach were important factors determining LOS (32). Even though the comparison between uniportal and multiportal VATS was not made the results are interesting. The Dutch healthcare system is similar to that in Sweden. Both countries have universal health care insurance coverage and easily accessible community health care facilities. Furthermore, the proportion of VATS lobectomies performed in the Netherlands in 2015 was similar to what was found at our institution during the same time period, $59 \%$ and $64 \%$, respectively (7). Short LOS does not automatically translate into less traumatic surgery or superior postoperative care, complications might occur after discharge. However, our institution is the sole provider of thoracic surgery in our region and when postoperative complications occur the absolute majority of the patients are readmitted at our hospital and the events registered in ThoR. Furthermore, all patients were followed at our outpatient clinic 4-6 weeks after surgery. A few previous studies have made the direct comparison of LOS between uniportal and multiportal VATS lobectomy and the results are conflicting. There are reports of an approximately one to two days decrease in LOS in patients operated on with uniportal VATS $(33,34)$, whereas others report of no significant difference in LOS related to surgical approach $(19,23,35)$, one study found an increase in LOS by 1.4 days in the uniportal group (36). These discrepancies might, however, be ascribed to differences in postoperative care routines regarding for example drain removal and discharge criteria. A recent systematic review and meta-analysis that aimed to determine and to give evidence-based recommendations regarding the optimal surgical approach for lobectomy reports of no significant difference in LOS between uniportal and multiportal VATS (18).

The majority of previous reports on chest drain duration comparing uniportal and multiportal VATS has not been able to show any significant differences related to surgical approach $(23,33,36,37)$. By contrast, Han et al. found a shorter chest drain indwelling time in the uniportal group (3.9 vs. 5.4 days) (38). In the present cohort the percentage of patients that had their chest drain removed on postoperative day one decreased over the study period and, in addition, was lower in uniportal group than in the multiportal group. The reason for this finding might be attributed to the fact that during the latter part of 2016 the Karolinska University Hospital moved in to new buildings and at the same time a new hospital organisation was implemented. This has caused some disturbances in terms of, for example, turnover of personnel at the ward which might have influenced the postoperative care. Furthermore, to remove the chest tube in patients who has undergone uniportal VATS might have been perceived as more challenging to the staff as the drain is placed into the surgical incision as opposed to in the camera port.

The low mortality and morbidity rates in this study are consistent with previous findings and are not surprising $(17,23,27)$. However, an important and still debated question is whether how, and to what extent, lymph nodes are harvested, affect survival, and if surgical approach is important in this context. Evidence-based guidelines regarding intraoperative lymph node resection are lacking but the Union for International Cancer Control (UICC) recommends that a minimum of six lymph nodes should be sampled from three or more N1 stations (hilar/ intrapulmonary) in addition to three or more N2 stations also including station seven $(15,39,40)$. Han et al. analysed 3 -year survival and recurrence-free survival at 3 -years in patients undergoing uni-, bi-, or multiportal VATS and found no significant survival differences related to surgical approach (38). There were no differences in the number of lymph nodes resected between the groups and according to the authors the dissection was routinely performed from all 
lymph node stations. There are other reports of no evident differences related to surgical approach with regard to extent of lymph node resection $(33,37)$ but also reports of a larger number of resected lymph nodes with a uniportal VATS approach $(34,41)$.

In our study the median number of lymph node stations sampled was four in the uniportal group throughout the study period. In the multiportal group the number increased to five stations over the years. The results are similar to what has been reported by $\mathrm{Mu} e t$ al. who found that the mean number of lymph node stations dissected with a uniportal approach was four as compared to six with a multiportal approach (36). The increase in the median number of lymph node stations dissected in the multiportal group during the study period might be attributed to an advancing skill set among the surgeons as they also acquired the technique of uniportal VATS. However, the proportion of patients that had more than three mediastinal lymph node stations sampled, including subcarinal nodes, was not in accord with UICC recommendations and, in addition, lower in the uniportal group than the multiportal group (40.2\% vs. $61.6 \%)$. Yet, subcarinal nodes were sampled to a very high extent in both groups (uniportal $91.8 \%$, multiportal $87.2 \%$ ), so whether this translates into a poorer long-term survival in this cohort is uncertain. In a study by Pawelczyk et al. the adherence to UICC recommendations was analysed in 3,376 patients undergoing surgery for a lung cancer and an, in total, adequate staging was found in $82.7 \%$ of the patients (42). The overall adequate staging improved from $62 \%$ in 2007 to $85 \%$ in 2017 . Adequate mediastinal sampling increased from $74 \%$ to $92 \%$ and sampling of subcarinal nodes increased from $75 \%$ to $93 \%$ over the years. Inadequate sampling was related to a significantly decreased survival. The factors influencing inadequate staging were identified as advanced patient age (likely due to a greater proportion of sublobar resections), sublobar resections (possibly due to a smaller quantity of resected and pathologically examined parenchyma, limited exploration of interlobar areas, and intention to minimize operating time and thus risk of complications), left sided resections (due to inadequate resection of mediastinal lymph nodes including subcarinal nodes), and small tumours. VATS was not found to be a factor that influenced inadequate staging.

Current evidence suggests that uniportal VATS is comparable to multiportal VATS in the surgical management of lung cancer, however, with the potential advantage of faster postoperative recovery (17). The present study corroborates this conclusion, though, it also underlines the importance of conducting institutional evaluations of the implementation of new surgical techniques and quality of surgery. Reported evidence of successfully implemented surgical approaches with preserved surgical and oncological quality is not necessarily generalizable to local settings. Future prospective, and preferably randomized, comparisons between minimally invasive approaches in lung cancer surgery are needed to truly detect clinically important differences in outcome.

\section{Study limitations}

This is a single institution, observational cohort study and as such has an inherent selection bias that cannot be completely eliminated with statistical methods of adjustment. Moreover, since the majority of the uniportal VATS lobectomies were performed by one single surgeon in combination with the single centre design limits the generalisability. However, all three surgeons were dedicated and experienced general thoracic surgeons and considered to be experts at the multiportal VATS lobectomy approach. Other limitations were the short follow-up, the lack of detailed information on the underlying mechanism for conversions and postoperative pain measurements, and the relatively small sample size preventing detection of possible differences between the groups regarding rare events.

\section{Conclusions}

We found that it was feasible and safe to implement uniportal VATS lobectomy into the treatment program of lung cancer patients at our institution. The results suggest that uniportal VATS lobectomy for lung cancer patients might entail advantages in terms of a faster recovery after surgery as compared to multiportal VATS lobectomy. Future studies with longer follow-up are needed to assure oncological efficacy.

\section{Acknowledgments}

The authors thank the ThoR steering committee for providing data for this study.

Funding: This work was supported by the Karolinska Institutet Foundations and Funds (grant number 201810530 to V Jackson); Swedish Heart-Lung Foundation (grant numbers 20160522, 20160525; and 20180400 to Dr U Sartipy); Åke Wiberg Foundation (grant number 2018: M18-0016 to Dr U Sartipy); Karolinska Institutet 
Foundations and Funds (grant number 2018-01784 to Dr U Sartipy); and the regional ALF agreement between Stockholm County Council and Karolinska Institutet (grant number 20180114 to Dr U Sartipy).

\section{Footnote}

Conflicts of Interest: The authors have no conflicts of interest to declare.

Ethical Statement: The authors are accountable for all aspects of the work in ensuring that questions related to the accuracy or integrity of any part of the work are appropriately investigated and resolved. The study was approved by the Swedish Ethical Review Authority and the need for informed consent was waived (Dnr: 2019-00964).

\section{References}

1. Yan TD, Cao C, D'Amico TA, et al. Video-assisted thoracoscopic surgery lobectomy at 20 years: a consensus statement. Eur J Cardiothorac Surg 2014;45:633-9.

2. Cao C, Frick AE, Ilonen I, et al. European questionnaire on the clinical use of video-assisted thoracoscopic surgery. Interact Cardiovasc Thorac Surg 2018;27:379-383.

3. Gonzalez D, Paradela M, Garcia J, et al. Single-port videoassisted thoracoscopic lobectomy. Interact Cardiovasc Thorac Surg 2011;12:514-5.

4. Kirby TJ, Rice TW. Thoracoscopic lobectomy. Ann Thorac Surg 1993;56:784-6.

5. Roviaro G, Rebuffat C, Varoli F, et al. Videoendoscopic pulmonary lobectomy for cancer. Surg Laparosc Endosc 1992;2:244-7.

6. Paul S, Isaacs AJ, Treasure T, et al. Long-term survival with thoracoscopic versus open lobectomy: propensity matched comparative analysis using SEER-Medicare database. BMJ 2014;349:g5575.

7. Al-Ameri M, Bergman P, Franco-Cereceda A, et al. Video-assisted thoracoscopic versus open thoracotomy lobectomy: a Swedish nationwide cohort study. J Thorac Dis 2018;10:3499-506.

8. Long H, Tan Q, Luo Q, et al. Thoracoscopic Surgery Versus Thoracotomy for Lung Cancer: Short-Term Outcomes of a Randomized Trial. Ann Thorac Surg 2018;105:386-92.

9. Yang CJ, Kumar A, Klapper JA, et al. A National Analysis of Long-term Survival Following Thoracoscopic Versus Open Lobectomy for Stage I Non-small-cell Lung Cancer.
Ann Surg 2019;269:163-71.

10. Falcoz PE, Puyraveau M, Thomas PA, et al. Videoassisted thoracoscopic surgery versus open lobectomy for primary non-small-cell lung cancer: a propensitymatched analysis of outcome from the European Society of Thoracic Surgeon database. Eur J Cardiothorac Surg 2016;49:602-9.

11. Laursen LØ, Petersen RH, Hansen HJ, et al. Videoassisted thoracoscopic surgery lobectomy for lung cancer is associated with a lower 30-day morbidity compared with lobectomy by thoracotomy. Eur J Cardiothorac Surg 2016;49:870-5.

12. Dziedzic R, Marjanski T, Binczyk F, et al. Favourable outcomes in patients with early-stage non-small-cell lung cancer operated on by video-assisted thoracoscopic surgery: a propensity score-matched analysis. Eur J Cardiothorac Surg 2018;54:547-53.

13. Bendixen M, Jorgensen OD, Kronborg C, et al. Postoperative pain and quality of life after lobectomy via video-assisted thoracoscopic surgery or anterolateral thoracotomy for early stage lung cancer: a randomised controlled trial. Lancet Oncol 2016;17:836-844.

14. Detterbeck FC, Lewis SZ, Diekemper R, et al. Executive Summary: Diagnosis and management of lung cancer, 3rd ed: American College of Chest Physicians evidence-based clinical practice guidelines. Chest 2013;143:7S-37S.

15. Postmus PE, Kerr KM, Oudkerk M, et al. Early and locally advanced non-small-cell lung cancer (NSCLC): ESMO Clinical Practice Guidelines for diagnosis, treatment and follow-up. Ann Oncol 2017;28:iv1-21.

16. Akter F, Routledge $\mathrm{T}$, Toufektzian L, et al. In minor and major thoracic procedures is uniport superior to multiport video-assisted thoracoscopic surgery? Interact Cardiovasc Thorac Surg 2015;20:550-5.

17. Sihoe ADL. Uniportal Lung Cancer Surgery: State of the Evidence. Ann Thorac Surg 2019;107:962-72.

18. $\mathrm{Ng} \mathrm{CSH,} \mathrm{MacDonald} \mathrm{JK,} \mathrm{Gilbert} \mathrm{S} \mathrm{et} \mathrm{al.} \mathrm{Optimal}$ Approach to Lobectomy for Non-Small Cell Lung Cancer: Systemic Review and Meta-Analysis. Innovations 2019;14:90-116.

19. Bertolaccini L, Viti A, Terzi A, et al. Geometric and ergonomic characteristics of the uniportal video-assisted thoracoscopic surgery (VATS) approach. Ann Cardiothorac Surg 2016;5:118-22.

20. Bedetti B, Bertolaccini L, Solli P, et al. Learning curve and established phase for uniportal VATS lobectomies: the Papworth experience. J Thorac Dis 2017;9:138-142.

21. Bertolaccini L, Rocco G, Pardolesi A, et al. P. The 
Geometric and Ergonomic Appeal of Uniportal Video-Assisted Thoracic Surgery. Thorac Surg Clin 2017;27:331-8.

22. Harris CG, James RS, Tian DH, et al. Systematic review and meta-analysis of uniportal versus multiportal videoassisted thoracoscopic lobectomy for lung cancer. Ann Cardiothorac Surg 2016;5:76-84.

23. Perna V, Carvajal AF, Torrecilla JA, et al. Uniportal videoassisted thoracoscopic lobectomy versus other videoassisted thoracoscopic lobectomy techniques: a randomized study. Eur J Cardiothorac Surg 2016;50:411-5.

24. Benchimol EI, Smeeth L, Guttmann A, et al. The REporting of studies Conducted using Observational Routinely-collected health Data (RECORD) statement. PLoS Med 2015;12:e1001885.

25. von Elm E, Altman DG, Egger M, et al. The Strengthening the Reporting of Observational Studies in Epidemiology (STROBE) statement: guidelines for reporting observational studies. J Clin Epidemiol 2008;61:344-9.

26. Hansen HJ, Petersen RH. Video-assisted thoracoscopic lobectomy using a standardized three-port anterior approach - The Copenhagen experience. Ann Cardiothorac Surg 2012;1:70-6.

27. Gonzalez-Rivas D, Paradela M, Fernandez R, et al. Uniportal video-assisted thoracoscopic lobectomy: two years of experience. Ann Thorac Surg 2013;95:426-32.

28. Hernandez-Arenas LA, Purmessur RD, Gonzalez-Rivas D. Uniportal video-assisted thoracoscopic segmentectomy. J Thorac Dis 2018;10:S1205-14.

29. Griffin BA, Ridgeway G, Morral A, et al. Toolkit for Weighting and Analysis of Non-equivalent Groups (TWANG) Website. 2014. Available online: http://www. rand.org/statistics/twang

30. Ridgeway G, McCaffrey D, Morral A, et al. twang: Toolkit for Weighting and Analysis of Non-equivalent Groups. 2017. Available online: https:/CRAN.R-project.org/ package=twang. $\mathrm{R}$ package version 1.5 .

31. Austin PC, Stuart EA. Moving towards best practice when using inverse probability of treatment weighting (IPTW) using the propensity score to estimate causal treatment effects in observational studies. Stat Med 2015;34:3661-79.

32. von Meyenfeldt EM, Marres GMH, van Thiel E, et al. Variation in length of hospital stay after lung cancer surgery in the Netherlands. Eur J Cardiothorac Surg 2018;54:560-4.

33. Chang JM, Kam KH, Yen YT, et al. From biportal to uniportal video-assisted thoracoscopic anatomical lung resection: A single-institute experience. Medicine (Baltimore) 2016;95:e5097.

34. Liu CC, Shih CS, Pennarun N, et al. Transition from a multiport technique to a single-port technique for lung cancer surgery: is lymph node dissection inferior using the single-port technique? Eur J Cardiothorac Surg 2016;49 Suppl 1:i64-72.

35. McElnay PJ, Molyneux M, Krishnadas R, et al. Pain and recovery are comparable after either uniportal or multiport video-assisted thoracoscopic lobectomy: an observation study. Eur J Cardiothorac Surg 2015;47:912-5.

36. Mu JW, Gao SG, Xue Q, et al. A Matched Comparison Study of Uniportal Versus Triportal Thoracoscopic Lobectomy and Sublobectomy for Early-stage Nonsmall Cell Lung Cancer. Chin Med J (Engl) 2015;128:2731-5.

37. Chung JH, Choi YS, Cho JH, et al. Uniportal videoassisted thoracoscopic lobectomy: an alternative to conventional thoracoscopic lobectomy in lung cancer surgery? Interact Cardiovasc Thorac Surg 2015;20:813-9.

38. Han KN, Kim HK, Choi YH. Midterm outcomes of single port thoracoscopic surgery for major pulmonary resection. PLoS One 2017;12:e0186857.

39. Rami-Porta R, Wittekind C, Goldstraw P, International Association for the Study of Lung Cancer Staging C. Complete resection in lung cancer surgery: proposed definition. Lung Cancer 2005;49:25-33.

40. International Association for the Study of Lung Cancer, IASCL Staging and Prognostic Factors Committee. Staging manual in thoracic oncology. Aurora (CO): International Association for the Study of Lung Cancer; 2016. 107-108 p.

41. Wang BY, Liu CY, Hsu PK, et al. Single-incision versus multiple-incision thoracoscopic lobectomy and segmentectomy: a propensity-matched analysis. Ann Surg 2015;261:793-9.

42. Pawelczyk K, Blasiak P, Szromek M, et al. Assessment of adequacy of intraoperative nodal staging and factors influencing the lack of its compliance with recommendations in the surgical treatment of non-small cell lung cancer (NSCLC). J Thorac Dis 2018;10:4902-11.

Cite this article as: Al-Ameri M, Sachs E, Sartipy U, Jackson V. Uniportal versus multiportal video-assisted thoracic surgery for lung cancer. J Thorac Dis 2019;11(12):5152-5161. doi: $10.21037 /$ jtd.2019.12.01 
Table S1 Baseline characteristics in patients who underwent multiport and uniport VATS lobectomy for lung cancer before and after inverse probability of treatment weighting

\begin{tabular}{|c|c|c|c|c|c|c|}
\hline \multirow{2}{*}{ Variable } & \multicolumn{3}{|c|}{ Unweighted } & \multicolumn{3}{|c|}{ IPTW } \\
\hline & Multiport & Uniport & SMD & Multiport* & Uniport* & SMD \\
\hline $\mathrm{n}$ & 211 & 122 & & 307.29 & 280.76 & \\
\hline Age, years, mean (SD) & $67.7(8.2)$ & $67.5(9.0)$ & 0.017 & $67.6(8.2)$ & $67.6(8.5)$ & 0.002 \\
\hline Female sex & $139(65.9)$ & $74(60.7)$ & 0.108 & $201.7(65.7)$ & $172.9(61.6)$ & 0.085 \\
\hline BMI, kg/m², mean (SD) & $25.6(4.5)$ & $25.3(4.2)$ & 0.073 & $25.4(4.4)$ & $25.4(4.4)$ & 0.011 \\
\hline Performance status $>0$ & $33(15.6)$ & $13(10.7)$ & 0.148 & $46.5(15.1)$ & $31.1(11.1)$ & 0.121 \\
\hline Smoking & & & 0.099 & & & 0.099 \\
\hline Never & $53(25.1)$ & $29(23.8)$ & & $77.1(25.1)$ & $60.1(21.4)$ & \\
\hline Former & $106(50.2)$ & $67(54.9)$ & & $155.0(50.4)$ & $153.8(54.8)$ & \\
\hline Current & $52(24.6)$ & $26(21.3)$ & & $75.2(24.5)$ & $66.8(23.8)$ & \\
\hline Preoperative FEV1, liter, mean (SD) & $2.30(0.61)$ & $2.39(0.77)$ & 0.137 & $2.31(0.62)$ & $2.35(0.71)$ & 0.062 \\
\hline No comorbidity & $94(44.5)$ & $73(59.8)$ & 0.310 & $145.4(47.3)$ & $150.4(53.6)$ & 0.125 \\
\hline Ischemic heart disease & $18(8.5)$ & $13(10.7)$ & 0.072 & $25.8(8.4)$ & $29.7(10.6)$ & 0.074 \\
\hline Hypertension & $106(50.2)$ & $48(39.3)$ & 0.220 & $145.8(47.5)$ & $115.1(41.0)$ & 0.130 \\
\hline Arrhythmia & $10(4.7)$ & $6(4.9)$ & 0.008 & $14.3(4.7)$ & $13.9(5.0)$ & 0.014 \\
\hline Prior thoracic surgery & $7(3.3)$ & $4(3.3)$ & 0.002 & $10.2(3.3)$ & $9.1(3.2)$ & 0.004 \\
\hline Congestive heart failure & $3(1.4)$ & $3(2.5)$ & 0.075 & $4.3(1.4)$ & $6.4(2.3)$ & 0.066 \\
\hline Diabetes & $29(13.7)$ & $14(11.5)$ & 0.068 & $40.2(13.1)$ & $37.4(13.3)$ & 0.007 \\
\hline Prior stroke/TIA & $14(6.6)$ & $7(5.7)$ & 0.037 & $20.2(6.6)$ & $19.9(7.1)$ & 0.021 \\
\hline Chronic kidney disease & $3(1.4)$ & $2(1.6)$ & 0.018 & $4.7(1.5)$ & $5.2(1.8)$ & 0.024 \\
\hline Preoperative radiotherapy & $3(1.4)$ & $1(0.8)$ & 0.057 & $3.9(1.3)$ & $1.9(0.7)$ & 0.063 \\
\hline Preoperative chemotherapy & $1(0.5)$ & $3(2.5)$ & 0.166 & $1.2(0.4)$ & $5.3(1.9)$ & 0.141 \\
\hline Preoperative positron emission tomography & $211(100.0)$ & $121(99.2)$ & 0.129 & $307.3(100.0)$ & $279.5(99.5)$ & 0.096 \\
\hline Perioperative lymph node sampling & $210(99.5)$ & $122(100.0)$ & 0.098 & $305.8(99.5)$ & $280.8(100.0)$ & 0.098 \\
\hline Pathologic stage & & & 0.214 & & & 0.124 \\
\hline IA & $100(47.4)$ & $52(42.6)$ & & $141.1(45.9)$ & $121.3(43.2)$ & \\
\hline IB & $60(28.4)$ & $41(33.6)$ & & $89.9(29.3)$ & $91.2(32.5)$ & \\
\hline$\| \mathrm{A}$ & $27(12.8)$ & $11(9.0)$ & & $38.1(12.4)$ & $28.9(10.3)$ & \\
\hline IIB & $14(6.6)$ & $8(6.6)$ & & $21.3(6.9)$ & $18.3(6.5)$ & \\
\hline IIIA-IV & $10(4.7)$ & $10(8.2)$ & & $16.8(5.5)$ & $20.9(7.5)$ & \\
\hline Postoperative histology & & & 0.048 & & & 0.082 \\
\hline Squamous & $18(8.5)$ & $11(9.0)$ & & $24.9(8.1)$ & $25.2(9.0)$ & \\
\hline Adenocarcinoma & $162(76.8)$ & 95 (77.9) & & $237.4(77.3)$ & 221.6 (78.9) & \\
\hline Carcinoid & $19(9.0)$ & $10(8.2)$ & & $28.2(9.2)$ & $20.2(7.2)$ & \\
\hline Other & $12(5.7)$ & $6(4.9)$ & & $16.8(5.5)$ & $13.7(4.9)$ & \\
\hline
\end{tabular}

Numbers are $\mathrm{n}(\%)$ unless otherwise noted. *, the overall numbers of patients in each group are not integers owing to inverse probability of treatment weighting. BMI, body mass index; FEV1, forced expiratory volume in one second; IPTW, inverse probability of treatment weighting; SD, standard deviation; SMD, standardized mean differences; TIA, transient ischemic attack; VATS, video-assisted thoracic surgery. 
Table S2 Number of lymph nodes sampled perioperatively and postoperative hospital stay

\begin{tabular}{lcc}
\hline & Multiport & Uniport \\
\hline Number of lymph node stations sampled & $5[4-5]$ & $4[4-5]$ \\
Postoperative hospital stay (days) & $4[3-4]$ & $3[2-4]$ \\
\hline
\end{tabular}

Numbers are median $\left(1^{\text {st }}\right.$ and $3^{\text {rd }}$ quartile).

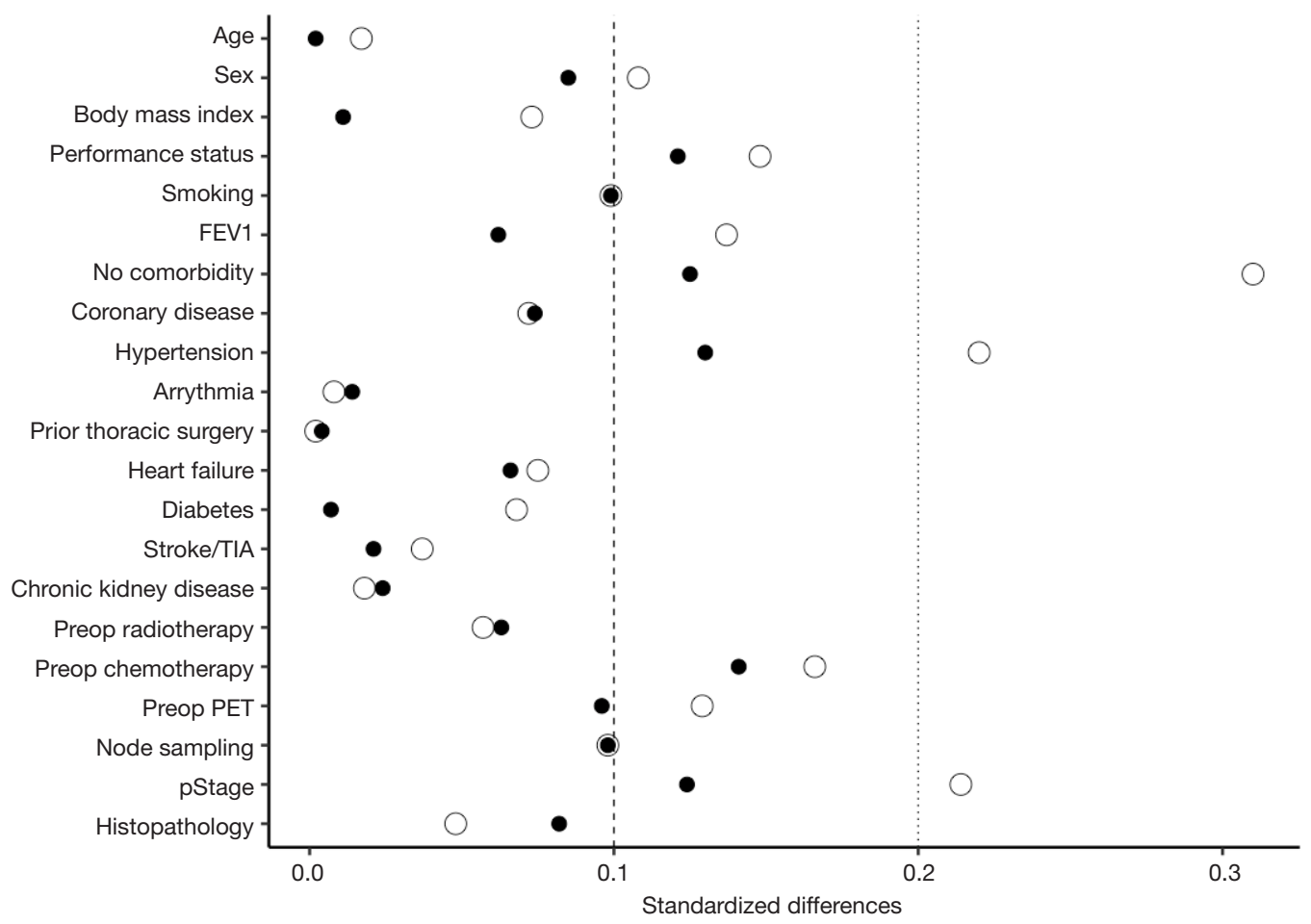

Figure S1 Absolute standardized differences before (hollow circles) and after (filled circles) inverse probability of treatment weighting. 